\title{
Multi-horn matching plate for ultrasonic transducers
}

\author{
Neville H. Fletcher* ${ }^{*}$ and Suszanne Thwaites \\ Division of Applied Physics, CSIRO, Bradfield Road, Lindfield, NSW 2070, Australia \\ † Research School of Physical Sciences and Engineering, Australian National University. \\ Canberra ACT 2601, Australia
}

Received 20 June 1991

The problem of matching a thick piezoelectric transducer to an air medium is considered and it is concluded that a suitable matching device can be designed and built in the form of a rigid plate, a few millimetres thick and pierced by a closely packed array of horn-shaped channels, the wide mouths of the horns facing the external world and the narrow throats facing the transducer. Design parameters, particularly the spacing between the multi-horn plate and the transducer surface, are found to be quite critical, but well within ordinary manufacturing capabilities. Methods of fabricating the multi-horn plates are described and measured results presented. The measured performance agrees quite well with design calculations. The experimental plates show a gain of $\approx 10 \mathrm{~dB}$ over a frequency range from $\approx 30$ to $100 \mathrm{kHz}$. Similar matching plates for other frequency ranges can be made.

Keywords: transducer; matching; impedance; horn

A common problem in the use of ultrasonic transducers in air is the very large impedance mismatch between the transducer and the medium. The wave impedance of a piezoelectric material such as barium titanate excecds that of air by a factor of $10^{5}$ and, even when the transducer is operated in a resonant mode, the effective acoustic mismatch is still of the order of $10^{3}$. The intrinsic mismatch for a non-resonant stretched-foil transducer is rather less but, without the assistance of resonance matching, the acoustic mismatch is still very large.

While better matching would improve power output when the transducer is used in the transmitting mode, the improvement in performance that could be achieved by better matching becomes particularly important in the receiving mode, particularly when signal levels are low. It is often not desirable to use a resonant transducer with very high quality factor $Q$, because of the associated degradation in time resolution.

These problems are, of course, not unique to air as a medium, nor to the ultrasonic frequency range, but the solutions that have been devised often apply only in other situations. Thus, for example, it is possible to use resonant (quarter-wave) matching layers with wave impedance logarithmically intermediate between that of the transducer and the medium for piezoelectric transducers operating at ultrasonic frequencies in water. It is difficult to find a low-loss material with the desired wave impedance for similar use in air. At low frequencies, on the other hand, horns provide a good measure of impedance matching, but horns operate efficiently over only a limited frequency range and are, as shown later, generally ineffective at ultrasonic frequencies. Paraboloidal or ellipsoidal reflectors

* To whom correspondence should be addressed do provide a universal remedy, but at the expense of large size.

A new approach to the impedance-matching problem in air at ultrasonic frequencies above $\approx 30 \mathrm{kHz}$ is described. The device is small, relatively simple to design and fabricate, works equally well with resonant and nonresonant transducers, and achieves an increase in gain of $>10 \mathrm{~dB}$ in typical cases.

\section{Device concept}

A horn is, from many points of view, a nearly ideal impedance transformer. Its lower cut-off frequency is determined by the flare rate of the horn profile and details of the cut-off behaviour by details of the profile $e^{1-3}$. Its upper cut-off frequency is determined by the total curvature of the wavefront at the horn mouth and thus by the mouth diameter and the semi-angle of the cone tangent to the horn surface ${ }^{3}$. Between these two cut-off frequencies, and assuming the throat to be terminated by a relatively high impedance, the pressure gain above the free-field value for a source on the axis is between 1 and 2 times the ratio of the mouth diameter to the throat diameter. The floating factor is 1 if the diameter of the horn mouth is small compared with the wavelength and 2 if it is large or if the mouth is surrounded by a baffle. Below the lower cut-off frequency the pressure gain tends to unity, while above the upper cut-off frequency the gain is small and strongly frequency dependent ${ }^{3}$.

The bandwidth of a horn increases, for given throat and mouth diameters, as its length increases, but this clearly leads to dimensional difficulties if the device is to be used in a confined space. Assuming a transducer diameter of, say, $10 \mathrm{~mm}$ and a desired pressure gain of 


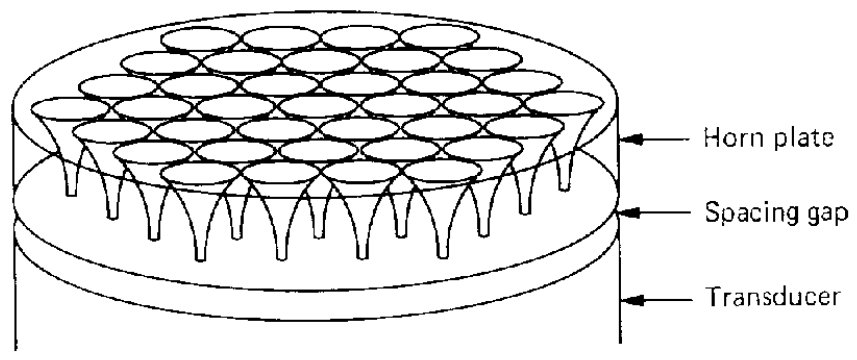

Figure 1 General structure of the proposed horn matching plate. A sealing ring closes the gap around its periphery

$\approx 10 \mathrm{~dB}$, a mouth diameter of $\approx 30 \mathrm{~mm}$ is required. (The floating factor is 2 for a horn of this size, but there is a balancing factor of 2 because the diameter of the transducer itself is large compared with the wavelength.) If the operating frequency is to be $100 \mathrm{kHz}$, then the length of the horn must exceed $\approx 100 \mathrm{~mm}$ in order to operate below the upper cut-off frequency ${ }^{3}$. At $200 \mathrm{kHz}$ the allowable wavefront curvature at the mouth is halved, so that the required horn length is doubled. (In neither case is the lower cut-off frequency the problem.)

The proposal is to replace this single horn by an array of much smaller horns, distributed over a plate which covers the surface of the transducer, as shown in Figure l. The transducer surface itself is separated by a very small distance from the side of the plate through which the throats of the small horns open. Each horn can be designed to operatc cfficiently at the desired high ultrasonic frequency, say $100 \mathrm{kHz}$, and effectively serves only the small area of transducer surface located close to its open throat. As shown below, an arrangement of this sort operating at $100 \mathrm{kHz}$ requires a plate no more than $2 \mathrm{~mm}$ in thickness covering the transducer surface and can readily give a pressure gain, and thus an increase in transducer output, of $10 \mathrm{~dB}$.

Before considering the design parameters of this device in detail, it is useful to sketch them in qualitative terms. The acoustic impedance of the transducer is usually so great that it is a reasonable approximation to treat both it and the solid material of the matching plate as being completely rigid. This simplifies the problem 10 a consideration of the pressure generated across the transducer surface by an axially incident sound wave. This must be compared with the value in the absence of the matching plate, which will be just twice the free-field pressure, since the transducer diameter is generally large compared with the operating wavelength. Each horn is effectively baffled by the surrounding matching plate. inserting a factor of 2 into its gain relative to the free field and balancing the floating factor of 2 referred to above.

It is possible to design a horn to achieve a large gain at a fixed frequency if all its dimensions are available for adjustment. However the individual horn mouths can be widened only until they form a close-packed array on the plate surface and the distance between horn axes will then be equal to their mouth diameters. Symmetry dictates that there is no acoustic flow across the hexagonal boundarics defining the two-dimensional cell associated with each horn throat and we can therefore treat each horn independently. It is clearly necessary for efficiency that there be only a small phase shift due to wave propagation in the space between the plate and the transducer surface in each cell. This sets the upper limit to the diameter of each cell at about a half wavelength and so sets the same limit on the horn-mouth diameter. For operation at a frequency of say $100 \mathrm{kHz}$, the wavelength is $\approx 3 \mathrm{~mm}$.

The diameter of the horn throat is set by a compromise between the desirability of a small diameter to give a large pressure gain in the horn and the desirability of a larger diameter to allow optimal matching to the space between the plate and the transducer surface. The nominal pressure gain of the horn, discussed above, refers to a situation in which the horn throat is rigidly blocked ${ }^{3}$ and of course this is not so in the present case. Attention must therefore be turned to the loading effect of the gap between the back face of the horn-plate and the front face of the transducer. The incident pressure excites standing waves in this space and they are damped both by motion of the transducer and, more importantly, by viscous and thermal losses at the two bounding surfaces. For optimal matching efficiency it is desirable to make the gap as thin as possible, conditional upon its width being much greater than the boundary-layer thickness. As will be shown, the boundary-layer thickness at the frequencies involved is less than $\approx 5 \mu \mathrm{m}$, so that the gap can be $\approx 20-50 \mu \mathrm{m}$, which is reasonable to achicve in practice. For matching of the cell to the horn, the area of the annular entry to the cell is required to be small compared with the area of the horn throat, which dictates a throat diameter that is preferably an order of magnitude greater than the gap height. For a frequency near $100 \mathrm{kHz}$, this implies a throat diameter of a few tenths of a millimetre. These dimensions lead us to expect an overall pressure gain of $\approx 10 \mathrm{~dB}$.

For horns as small as this, the dimensional limits are set by the lower rather than the upper cut-off frequency, and, for the diameter ratio suggested above, the horns need to be about half a wavelength long. For a lower frequency limit of $30 \mathrm{kHz}$, this gives a matching-plate thickness of a few millimetres, which is mechanically convenient.

This brief outline shows that the essentials of the proposed device are simple and that it should be possible to optimize the available parameters to produce useful gain without occupying appreciable space in the vicinity of the transducer.

\section{Detailed analysis}

The conceptual outline above has identificd several areas for which detailed analysis is required. These are the horn dimensions and profile, and the behaviour of the space between the matching plate and the transducer surface. These will be dealt with in turn and it will be shown how they can be optimized.

\section{Behaviour of the horn}

The device described has a horn, the mouth of which is open to an axially incident sound field and with a throat terminated by an impedance which is yet to be defined. To solve this problem in detail and to optimize the horn configuration, analytical results are required for a family of horns whose profiles can be varied over a reasonably wide range. Although there are analytical solutions for infinite horns having the geometry of a hyperboloid of one sheet, no exact analytical solutions exist for widemouthed horns of linite length. Therefore horns are considered in the usual approximation represented by 

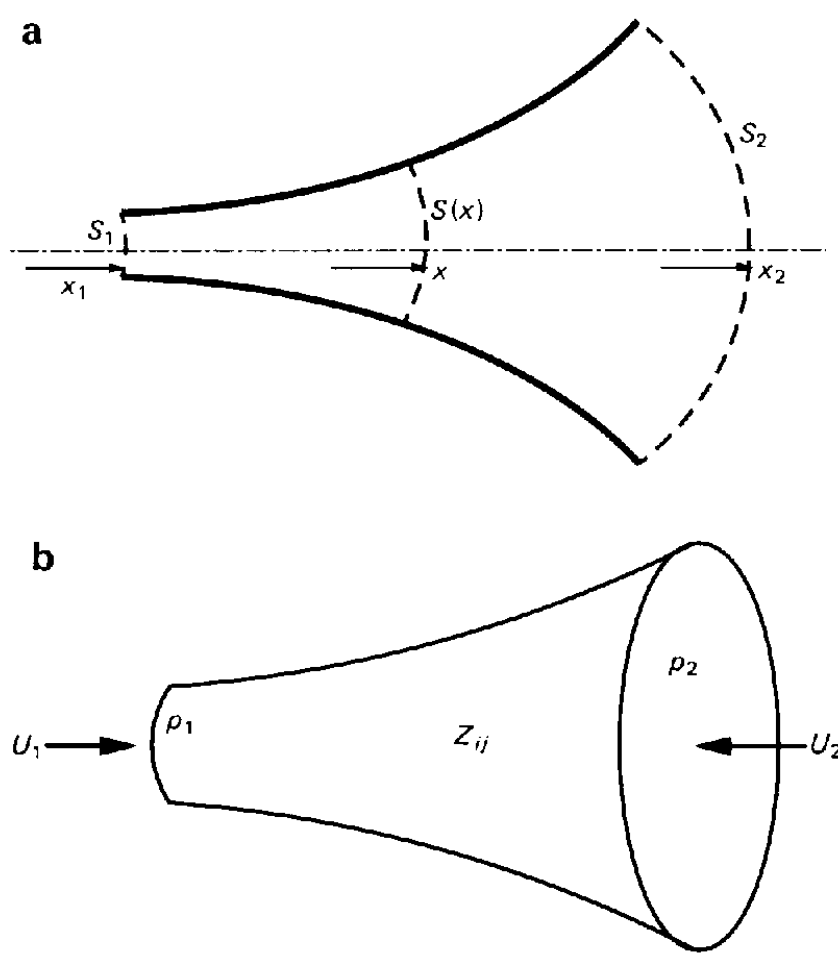

Figure 2 Parameters for the horn behaviour. (a) Coordinates and wavefronts for the horns discussed in the text. (b) Definition of acoustic quantities for a general horn, leading to the impedance coefficients $Z$

the Webster equation ${ }^{1}$

$\frac{1}{S} \frac{\partial}{\partial x}\left(S \frac{\partial p}{\partial x}\right)=\frac{1 \partial^{2} p}{c^{2} \partial t^{2}}$

where $x$ is the axial coordinate giving the wavefront position in the horn as shown in Figure $2 a, S(x)$ is the area of the curved wavefront surface within the horn, $p(x)$ is the acoustic pressure and $c$ is the normal free-field speed of sound. This equation represents an adequate approximation ${ }^{4,5}$ for the present case, but there are some difficulties associated with the curvature of the wavefronts in the horn that are generally overlooked ${ }^{3,6}$. The possibility of higher modes within the horn is ignored because, in the cases involved, the horn diameter is so small that such higher modes are rapidly attenuated.

There are two families of horns which have reasonably wide ranges of profiles and for which the analytical solution of (1) is not too difficult. The first of these is the family of Salmon or 'hypex' horns ${ }^{1,2,5}$ and the second is the family of Bessel horns ${ }^{6}$. Between them these horns range from cup-shaped through conical to widely flaring. In practice, in the present application, the family of exponential horns, a sub-set of the hypex family, provides adequate acoustic flexibility for our purpose, so attention will be confined to such horns.

Most texts, for example Morse ${ }^{1}$ and Olson ${ }^{2}$, simply solve the Webster equation (Equation 1) to determine the input impedance at the throat of the horn when its mouth is terminated by the normal radiation impedance. While this simple result is a useful guide, more detailed information is required in the form of the impedance coefficients $Z_{i j}$, defined by the equations

$p_{1}=Z_{11} U_{1}+Z_{12} U_{2}$

$p_{2}=Z_{21} U_{1}+Z_{22} U_{2}$ where $U_{1}$ and $U_{2}$ are acoustic volume flows into the throat and mouth respectively, as defined in Figure $2 b$. Some authors use other conventions, with consequent changes of sign in the impedance quantities. Clearly the $Z_{i j}$ will depend upon the wavefront areas $S_{1}$ and $S_{2}$ at the throat and mouth respectively, upon the horn length $l=x_{2}-x_{1}$ and upon the parameters determining the horn profile. These impedance coefficients have been given for paraboloidal, conical and exponential horns by Fletcher and Thwaites ${ }^{3}$, and it is simple to derive corresponding relations for the Salmon horn family.

In the expressions that follow, and in all the subsequent development, the physics convention is used of representing time variation by the complex exponential $\exp (-i \omega t)$, rather than the electrical engineering convention $\exp (j \omega t)$. For those more familiar with the latter convention, all formulae can be directly rewritten by replacing $i$ by $-j$.

For an exponential horn the flare constant $m$ is defined by

$m=\frac{1}{2 l} \ln \left(\frac{S_{2}}{S_{1}}\right)$

and further defined by

$b=\left(k^{2}-m^{2}\right)^{1 / 2}, \quad \theta=\tan ^{-1}(m / b)$.

It turns out that the major acoustic attenuation in the system occurs in the narrow space between the matching plate and the transducer, so that it is an adequate approximation to neglect wall losses in the horn and to take $k$ to be the real quantity $\omega / c$. The impedance coefficients then have the explicit forms

$\mathrm{Z}_{11}=\frac{i \rho c}{S_{1}}\left[\frac{\cos (b l+\theta)}{\sin (b l)}\right]$
$\mathrm{Z}_{22}=\frac{i \rho c}{S_{2}}\left[\frac{\cos (b l-\theta)}{\sin b l}\right]$
$\mathrm{Z}_{12}=\frac{i \rho c}{\left(S_{1} S_{2}\right)^{1 / 2}}\left[\frac{\cos \theta}{\sin b l}\right]$.

The cut-off frequency is given by

$\omega^{*}=m c, \quad f^{*}=m c / 2 \pi$.

For $f<f^{*}$, both $b$ and $\theta$ become imaginary, so that the expressions for the $Z_{i j}$ need to be rewritten in terms of hyperbolic functions before evaluation.

Assuming that the coefficients $Z_{i j}$ are known, an expression is needed for the acoustic pressure developed in the throat of the horn when its open mouth is exposed to an axially incident sound wave with free-ficld pressure $p_{0}$ and the throat is terminated by an acoustic impedance $Z_{\mathrm{C}}$, corresponding to the input impedance of the cell between the plate and the transducer, which will be calculated in the next section. The network for this situation is shown in Figure 3. Within the one-dimensional formalism adopted, the plane-wave source behaves like a Thévenin generator of pressure $2 p_{0}$ and internal impedance equal to the radiation impedance $Z_{\mathrm{R}}$ at the horn mouth, as discussed by Fletcher and Thwaites ${ }^{3}$. It should be noted, however, that the radiation impedance at the mouth of an individual horn is influenced by the presence of neighbouring horn mouths. The radius, $A$ say, of the horn array itself is generally large enough that $k A \gg 1$ at the operating frequency, so that the mechanical load of the radiation impedance is very nearly the simple 


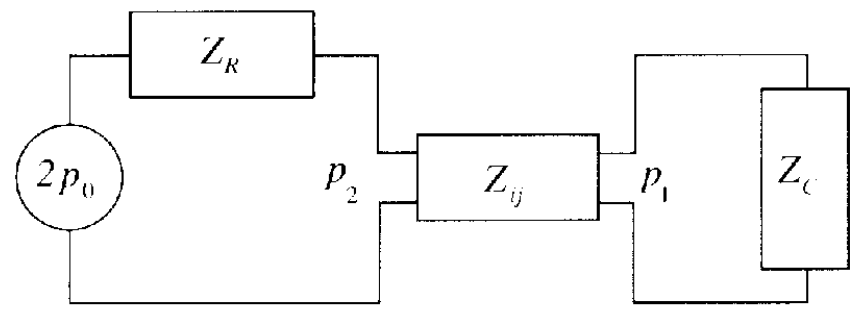

Figure 3 Network illustrating the situation in which a large or baffled horn is exposed to an axially incident free-field acoustic pressure $p_{0}$ and connected to an acoustic load $Z_{C}$ at its throat

resistive quantity $\rho c$ per unit area and the reactive component is very small. When divided between the component small horns, this gives each an acoustic radiation resistance $\rho c / \pi a^{2}$ and nearly no reactive component or end correction.

From Figure 3 and Equations (2) and (3) the acoustic pressure $p_{1}$ in the horn throat is related to the free-ficld pressure po by

$\frac{p_{1}}{2 p_{0}}=\frac{Z_{12} Z_{\mathrm{C}}}{\left(Z_{\mathrm{R}}+Z_{22}\right)\left(Z_{\mathrm{C}}+Z_{11}\right)-Z_{12}^{2}}$

This quantity has a set of maxima at the frequencies for which the denominator goes through a minimum. corresponding to standing waves in the horn and its terminating cavity. The lowest of these would be a sort of Helmholtz. resonance if the cavity were large enough. but in fact the cavity impedance is so large that the resonance frequencies are very nearly those of the horn when blocked at its throat. These resonance are, however. highly damped because the horn mouth is large enough relative to the wavelength that the resistive component of the radiation impedance $Z_{\mathrm{R}}$ is very nearly equal in magnitude to the average value of $Z_{22}$

\section{Behaviour of the spacing gap}

The geometry of a single cell of the gap between the matching plate and the transducer surface is shown in Figure 4. For the assumed close-packed horn array, the cell is hexagonal, but for simplicity of analysis this has been replaced by a circular cell of equal area. The cell radius $a^{\prime}$ is then equal to $1.05 a$, where $a$ is the horn-mouth radius. It is assumed that the height $d$ of the cell. corresponding to the spacing between matching plate and transducer, is very much smaller than the cell radius ${ }^{\prime}$ '. The inner radius $b$ of the cell is equal to the radius of the horn throat, and it is assumed that $b \ll a$.

There will be thermal and viscous losses to the plane walls of the cell across the boundary layers, which must be laken into account, but, provided the cell height $d$ is greater than the boundary layer thickness $\delta$, this will simply lead to an attenuation coefficient for propagating waves. The situation is not very different, simply more extreme, if $d<\delta$. The acoustic wavefronts in the cell have the form of circular cylinders, though losses contribute a variation in amplitude across the height of the cell. If $r$ is the radial coordinate, then the solution to the wave equation in cylindrical polar coordinates is

$p(r) \div A^{\prime} H_{0}^{(1)}(k r)+B^{\prime} H_{0}^{(2)}(k r)=A J_{0}(k r)+B N_{0}(k r)$

where $A^{\prime}$ and $B^{\prime}$ are complex constants and $H_{0}^{(1)}$ and $H_{0}^{(2)}$ are respectively Hankel functions of the first and second $k$ inds, representing outgoing and incoming waves. In the second form of writing. $A$ and $B$ are again complex constants, and $J_{m}$ and $N_{m}$ are respectively Bessel functions and Neumann functions. (The Neumann functions are sometimes ${ }^{7}$ denoted by $Y_{\mathrm{m}}$, but this can lead to confusion with spherical harmonics.) From symmetry

$\left[\begin{array}{l}\hat{p} \\ \hat{i r}\end{array}\right]_{r} \quad-0$

which fixes the ratio $B: A$ and gives

$p(r)-A\left[J_{0}(k r)-\frac{J_{1}\left(k a^{\prime}\right)}{N_{1}\left(k a^{\prime}\right)} N_{0}(k r)\right]$

The acoustic volume flow $U(r)$ is given by

$U(r)-\left(\frac{2 i \pi r d}{\rho(t)}\right) i r$

so that the acoustic impedance at the inner radius where the annular cell connects to the horn throat $r=h$ is

$L_{c}=\frac{i p c}{2 \pi b d}\left[\frac{J_{0}(k b) N_{1}\left(k a^{\prime}\right)-J_{1}\left(k a^{\prime}\right) N_{0}(k b)}{J_{1}(k b) N_{1}\left(k a^{\prime}\right)} \cdot \frac{J_{1}\left(k a^{\prime}\right) N_{1}(k b)}{]}\right]$

To lake account of the important effects of losses to the planar cell walls across the viscous and thermal boundary layers, the propagation constant $k$ must be treated as a complex quantity. The essential details are given by Back us ${ }^{8}$. The thicknesses $\delta_{\mathrm{v}}$ and $\delta_{1}$ of the viscous and thermal boundary layers are given by

$$
\begin{aligned}
& \left.j_{v}=(1)(1) p\right)^{1.2} \approx 3.9 \times 10^{-3}, 12 \text {, } \\
& \delta_{1}-\left(K(2)\left(C_{n}\right)^{12} \approx 4.7 \times 10^{3}{ }^{3} \cdot{ }^{\prime \prime}\right)^{2}
\end{aligned}
$$

where $\eta$ is the viscosity, $K$ the thermal conductivity. $C_{p}$ the specific heat at constant pressure, and $\rho$ the density of the gas in the space. The numerical values are for air at $23 \mathrm{C}$.

Backus ${ }^{8}$ gives expressions for the series impedance $Z$ and shunt admittance $Y_{\mathrm{p}}$ for propagation between two parallel planes separated by a distance $d$. The propagation constant $k=-i\left(Z_{\mathrm{s}} Y_{\mathrm{n}}\right)^{1 / 2}$ takes on the complex value $\left(a c^{\prime}\right)+i x$ and it is most convenient here simply to give numerical approximations to the final results, which are algebraically complicated.

The sound speed $c^{\prime}$ for propagation between two plates in air is given to an adequate approximation by the

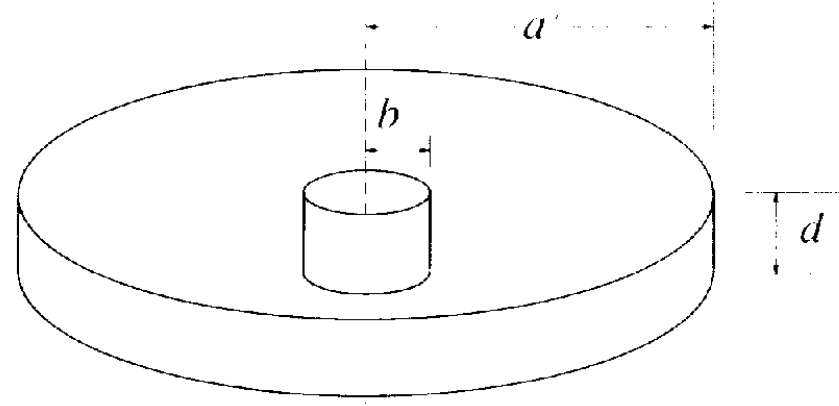

Figure 4 Simplified geometry for are of the cells between the horn plate and the transducer surface. The true hexagonal cell has been replaced by a circular cell of the same area 

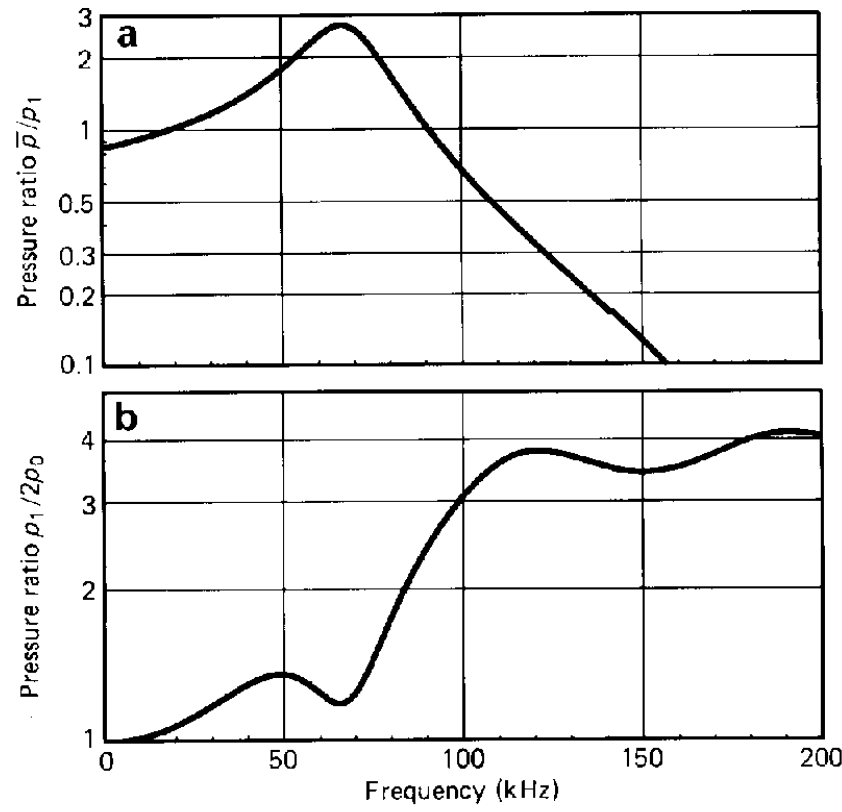

Figure 5 Pressure calculations. (a) Average pressure ratio $\bar{p} / p_{1}$ across the surface of the transducer for a cell radius $a=1 \mathrm{~mm}$, fed by a pressure $p_{1}$ applied to a central port of radius $b=0.25 \mathrm{~mm}$, as a function of frequency. (b) Pressure $p_{1}$ in the horn throat, relative to twice the free-field pressure $p_{0}$ for the design parameter values given in Table 1

empirical fitting formula

$c^{\prime} / c \approx\left(1-1.2 e^{-140 d\left(3^{1 / 2}\right)}\right)\left(1-0.1 e^{-15 d()^{1 / 2}}\right)+0.18 e^{-500 d(2)^{1 / 2}}$

over the whole range in which we are interested. For air at room temperature the attenuation coefficient $\alpha$ can similarly be fitted by the empirical relation

$\alpha \approx 1.2 \times 10^{5}\left(\omega^{1 / 2} / d\right)\left[1-0.4 \tanh \left(20 d^{1 / 2} \omega^{1 / 4}-2.5\right)\right]$

in units of $\mathrm{m}^{-1}$. The attenuation is essentially proportional to $\omega^{1 / 2} / d$ and the following bracketed function serves simply to change the slope part-way through the range.

It should be remembered that this is the behaviour appropriate for walls that are smooth on the scale of the boundary layer. The experimental devices to be described later had surface roughness of $\approx 5 \mu \mathrm{m}$, which is comparable with the boundary layer thickness at frequencies above $\approx 100 \mathrm{kHz}$. For frequencies significantly higher than this, surface effects are expected to become more severe by perhaps as much as a factor of 2 , depending on the surface roughness, corresponding to the larger effective surface area involved. Calculations show that the change this would produce in the gain curve is not large and indeed the reduced cancellation produced by outer counter-phase zoncs on the plate paradoxically tends to increase the gain in those regions where it is much less than $0 \mathrm{~dB}$, while having little effect when the gain is high. This refinement is omitted in the following discussion.

The resulting Equations (13), (14) and (15) must all be evaluated with the complex nature of $k$ in the spacing gap in mind. This complicates the evaluation considerably, but is essential since this is the major source of damping in the device.

From the general concept outlined in the introduction, it is clear that the device will work efficiently only if the propagation loss between the centre and the edges of the cells is small, so that $\alpha a^{\prime}<1$. From Equation (18) and at a frequency of the order $100 \mathrm{kH} z, d>a^{\prime} / 100$ is required to meet this criterion. This suggests a lower limit of about $a^{\prime} / 30$, which is typically some tens of microns and is thus reasonable from the point of view of fabrication.

Since all parts of the transducer are electrically in parallel (although one can imagine complicated electrode arrangements for which this is not true), the effective acoustic quantity driving the transducer is the average pressure over the cell area. If $p_{1}$ is the pressure in the throat of the horn, then the average pressure on the transducer surface is

$\bar{p}=\frac{b^{2}}{a^{\prime 2}} p_{1}+\frac{2}{a^{\prime 2}} \int_{b}^{a} p(r) r \mathrm{~d} r$

If the imaginary part of $k$ is not too large, this average can be evaluated from Equation (13), using the results (11.3.20) and (11.3.24) from Abramowitz and Stegun?, which yield

$$
\begin{aligned}
& \int_{b}^{a \prime} J_{0}(k r) r \mathrm{~d} r=\frac{1}{k^{2}}\left[k a^{\prime} J_{1}\left(k a^{\prime}\right)-k b J_{1}(k b)\right] \\
& \int_{b}^{a r} N_{0}(k r) r \mathrm{~d} r=\frac{1}{k^{2}}\left[k a^{\prime} N_{1}\left(k a^{\prime}\right)-k b N_{1}(k b)\right]
\end{aligned}
$$

and then making a Taylor expansion of the Bessel and Neumann functions about their values on the real axis.

The resulting average pressure $\bar{p}$, in the form $\bar{p} / p_{1}$, is shown as a function of frequency in Figure $5 a$, for typical values of the horn diameter and plate spacing, as given in Table 1. The damped cylindrical-wave resonance peak is clearly visible, as also is the decline at high frequencies caused by anti-phase zones. Good matching between the cell and the transducer requires that the frequency should be less than $\approx 100 \mathrm{kHz}$ in this case, corresponding to $k a^{\prime}<2$. There would clearly be advantage in operating the device near the frequency of the cavity resonance, if this does not adversely affect matching to the horn.

\section{Matching of horn and cavity}

In discussing the device concept, it was suggested that matching of the cavity to the horn requires that the magnilude of the cavity input impedance $Z_{C}$ should be large compared with the throat impedance

$Z_{\mathrm{H}}=Z_{11}-\frac{Z_{12}^{2}}{Z_{\mathrm{R}}+Z_{22}}$

of the horn. This imposes limits on the throat radius $b$ and the cavity height $d$, as we have already discussed qualitatively.

Figure $5 b$ shows the general behaviour of pressure in the horn throat as a function of frequency for a horn with the parameters given in Table 1. The dip in the curve

Table 1 Typical design parameters

\begin{tabular}{|lll|}
\hline Parameter & Symbol & Size \\
\hline Horn mouth diameter & $2 a$ & $2 \mathrm{~mm}$ \\
Horn throat diameter & $2 b$ & $0.5 \mathrm{~mm}$ \\
Horn cut-off frequency & $f^{*}$ & $30 \mathrm{kHz}$ \\
Horn length & 1 & $2.5 \mathrm{~mm}$ \\
Spacing gap width & $d$ & $30 \mu \mathrm{m}$ \\
\hline
\end{tabular}


near $70 \mathrm{kHz}$ is caused by the admittance maximum of the cavity at its resonance. The heavy radiation damping at the horn mouth suppresses the effects of horn resonances. The overall performance of the system is obtained essentially by multiplying the effective horn gain of Figure $5 b$ by the cavity gain of Figure $5 a$. The easiest way to optimize the design, as far as this matching is concerned, is to carry out a series of numerical calculations in which the relevant parameters are varied systematically around the optimum values expected from the qualitative discussion of the device concept. The results of such an optimization are considered below, since it must be carried out in conjunction with optimization of other parts of the system.

\section{Design optimization}

Optimization of the design within the framework defined involves four parameters - the horn mouth and throat radii $a$ and $h$, the horn length $l$ or cut-off frequency $f^{*}$, and the plate spacing $d$. Also available is the detailed form of the horn profile, should such refinement be necessary. It is convenient to take the mouth diameter of the horn as establishing the general physical scale and then to determine the best operating frequency for the resulting optimized design. This result can then be scaled and recalculated for the desired operating frequency.

The initial stages of a numerical analysis of the behaviour of the device indicate that the horn should generally operate substantially above its lower cut-off frequency in order to achieve best results. In this frequency range there is little difference between the behaviour of horns with different hypex profiles (as long as they are not conical) and it is therefore convenient to concentrate upon the exponential horn, for which expressions have already been given for the impedance coefficients. Because of heavy radiation damping at the horn mouth, the infinities and zeros in these impedance coefficients have very little effect upon the behaviour of the horn.

The curves shown in Figure 5 are partial results on the way to optimization of the whole design and figures similar to these can be used to investigate the effect of changing some of the parameters. Optimization of the whole design involves varying the accessible parameters so as to maximize the value of the ratio $G=\bar{p} / 2 p_{0}$ between the average pressure $p$ acting on the transducer surface under the matching plate and the pressure $2 p_{0}$ that would have acted on that surface in the absence of the plate, the diameter of the transducer being much greater than the acoustic wavelength. The quantity $G$ is the gain of the plate, and is conveniently expressed in decibels.

The design parameters given in Table $l$ refer to a matching plate optimized in this way, as do the partial results shown in Figure 5 . The calculated gain curve $G(f)$ for the system is shown in Figure 6. From this it is seen that the best operating frequency for this matching device is between 60 and $80 \mathrm{kHz}$ and that in this range it is expected to achieve a gain of $\approx 10 \mathrm{~dB}$. If the frequency range for calculation is extended to $400 \mathrm{kHz}$, then the response shows another maximum at $\approx 300 \mathrm{kHz}$, as expected, but its magnitude is insufficient to bring the system gain up to $0 \mathrm{~dB}$, so that it is of no practical utility.

\section{Matching plate fabrication}

An obvious good feature of the multi-horn plate is that it lends itself to fast and cheap manufacture. Since the

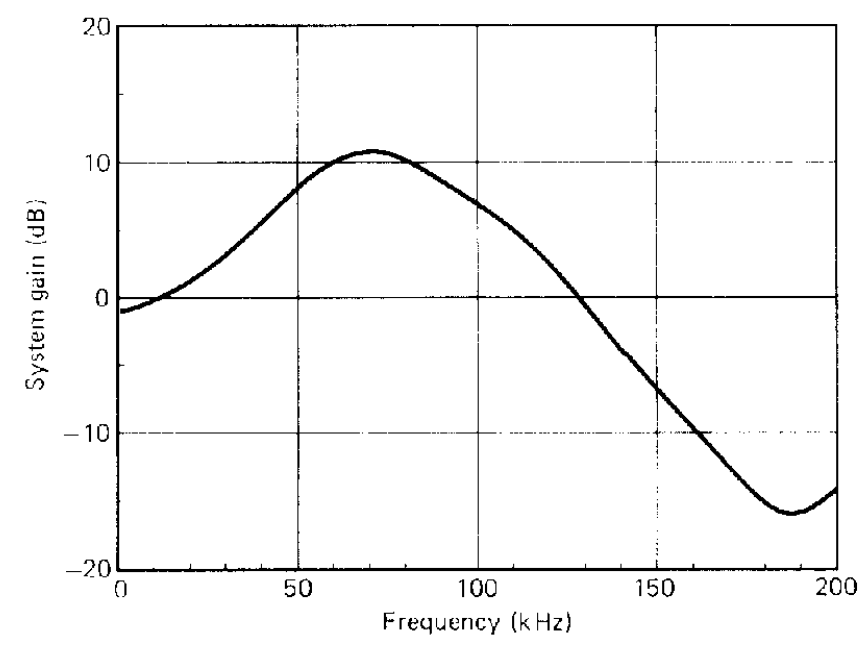

Figure 6 Calculated gain of the horn matching plate, with physical parameters given in $T a b / c 1$, as a function of frequency

constituent material is only required to be acoustically dense compared to air, great flexibility is allowed in its choice and the optimum dimensions lend themselves to fabrication by injection moulding. In the laboratory, however, individual plates need to be made with different characteristics without recourse to elaborate production methods.

The experimental plates were cast using a casting araldite. To make the moulds, the individual horn shapes were turned from narrow diameter brass rods using a numerically controlled jeweller's lathe. These pins had the horn shape at one end and a residual cylindrical section $=10 \mathrm{~mm}$ long. A bundle of the pins, coated with teflon mould release, was held tightly in a teflon ring machined to a hexagonal shape, with the horn-shaped points located against a flat base. With this encouragement the pins readily organized thmselves into a close packed structure. After casting and curing, the pins were easily removed to release the semi-completed plate. Figure 7 illustrates this procedure.

After initial flattening of both sides of the plate in a lathe, various forms of finishing technique were tried, ranging from simple lapping to the use of an optical finish grinder. Trial and crror methods were required to obtain a satisfactory compromise between surface roughness and flatness. Values for both quantities of $\approx 5 \mu \mathrm{m}$ were achieved across the surface of the plate, which is adequate, though closer tolerances are desirable.

Several different sets of plates were made, using pins shaped to give different horn cut-off frequencies. Within each set it was also easily possible to vary the throat diameter by changing the amount turned off the throat side of the plate during finishing. Throat diameters were measured using a profile projector and a uniformity to within better than $5 \%$ across the plate was typically achievable, though the small throats were often somewhat less smooth in aperture shape. In this way, adequate variation of parameters was achieved for experimental investigation of the design. Results are reported below for a representative design with the parameters given in Tuble 2 .

\section{Verification of performance}

To evaluate performance, the plates were used with it PZT-4 transducer $12 \mathrm{~mm}$ thick and $30 \mathrm{~mm}$ in diameter, 
a

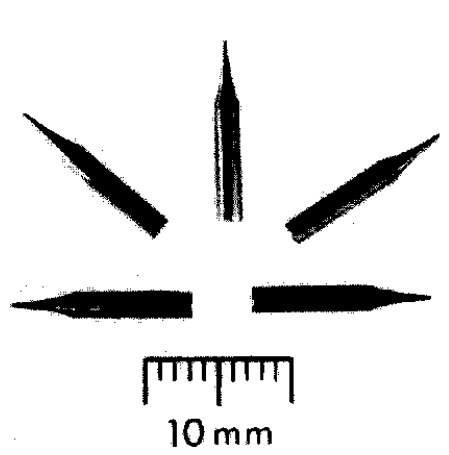

b

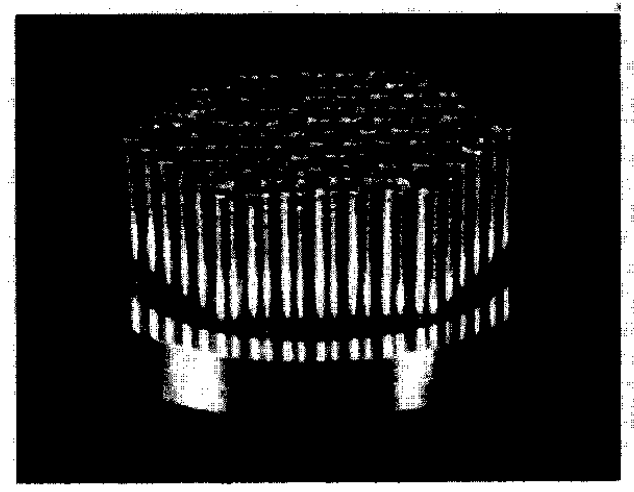

C

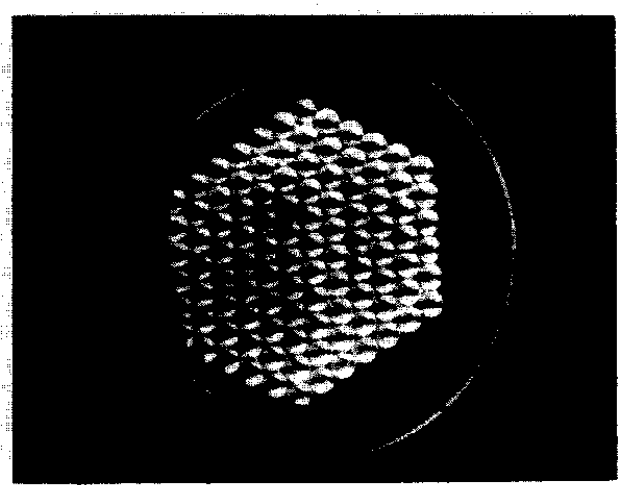

Figure 7 (a) Pins for horn casting; (b) the pin assembly during casting; (c) a finished multi-horn plate

Table 2 Details of experimental multi-horn plate

\begin{tabular}{|ll|}
\hline Parameter & Size \\
\hline Plate diameter & $22 \mathrm{~mm}$ \\
Initial plate thickness & $4.5 \mathrm{~mm}$ \\
Horn mouth diameter & $2.0 \mathrm{~mm}$ \\
Initial horn throat diameter & $0.34 \mathrm{~mm}$ \\
Horn cut -off frequency & $22 \mathrm{kHz}$ \\
\hline
\end{tabular}

the exposed edges being shielded. This transducer had five useful resonances below $200 \mathrm{kHz}$, of which the lowest was a radial mode at $60 \mathrm{kHz}$. The transducer was mounted in a case, making electrical contact to the back, and a fine wire contact was attached to the rim of the front in such a way that it did not interfere with positioning the multi-horn plate. The transducer was driven from the output of a HP-4194 gain-phase analyser and the acoustic signal was detected with a $1 / 8$ in condenser microphone, $B \& K 4180$, the response of which was flat to within $1 \mathrm{~dB}$ from $2-100 \mathrm{kHz}$, positioned about $12 \mathrm{~cm}$ in front of the plate. The microphone was equipped with a conical screen to eliminate reflections.

In order to vary the spacing gap $d$, the transducer was mounted on a table having a microscope traversing mechanism and the multi-horn plate was mounted rigidly. In this way the transducer-plate spacing could be continuously varied with a resolution of $2-3 \mu \mathrm{m}$. This apparatus is shown schematically in Figure 8.

For most of the measurements the transducer and plate were used as the transmitter but, when used as a receiver, a second transducer of the same type acted as the sound source. All the readings were normalized against the output of the transducer with a hexagonally shaped mask, of the same area as the active area of the horn plate, placed in front of it. Comparison then gave a direct measure of the gain. Such a procedure was essential, since the high $Q$ of the transducer rendered its acoustic output highly variable with respect to frequency. The results are all given as the ratio of the detected sound pressure with the horn plate to that of the same quantity with the mask, $p_{\mathrm{H}} / p_{\mathrm{M}}$. This ratio is identical to the pressure gain $G$ discussed in the theory, and the reciprocity theorem guarantees that it applies to the use of the matching plate in both receiving and transmitting configurations.

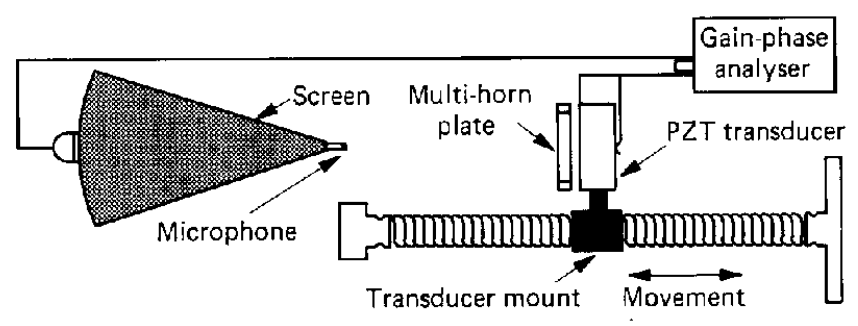

Figure 8 Apparatus used in measurement of multi-horn plate pressure gain

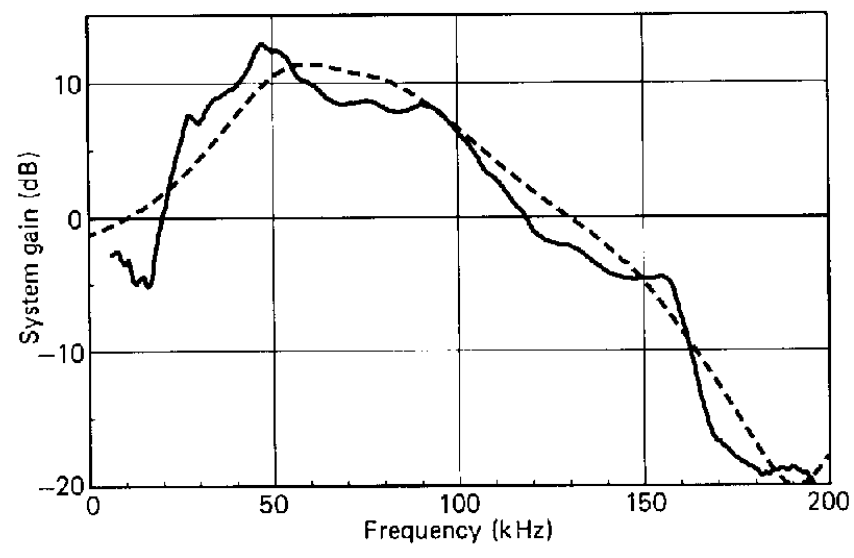

Figure 9 Measured sound pressure gain as a function of frequency for the design in Table 2. The broken curve shows the behaviour calculated from the theory

\section{Frequency response}

The frequency response of the transducer plus matching plate was measured between 2 and $200 \mathrm{kHz}$ using the swept frequency of the gain-phase analyser. The spacing gap was adjusted to give maximum response at $60 \mathrm{kHz}$ in each case, as will be described below. Typical measured results are shown in Figure 9. Superimposed on these is the response calculated from the model. For the theoretical curve the assumed gap spacing was $d=40 \mu \mathrm{m}$, a best estimate for the experimental value.

The frequency response of the PZT transducer was not very satisfactory at the higher frequencies, being a series of sharp resonances, but very careful measurements involving long integration times and a great deal of averaging left the results unchanged. Measurements with the microphone shifted 5 or $10 \mathrm{~mm}$ from the axial 


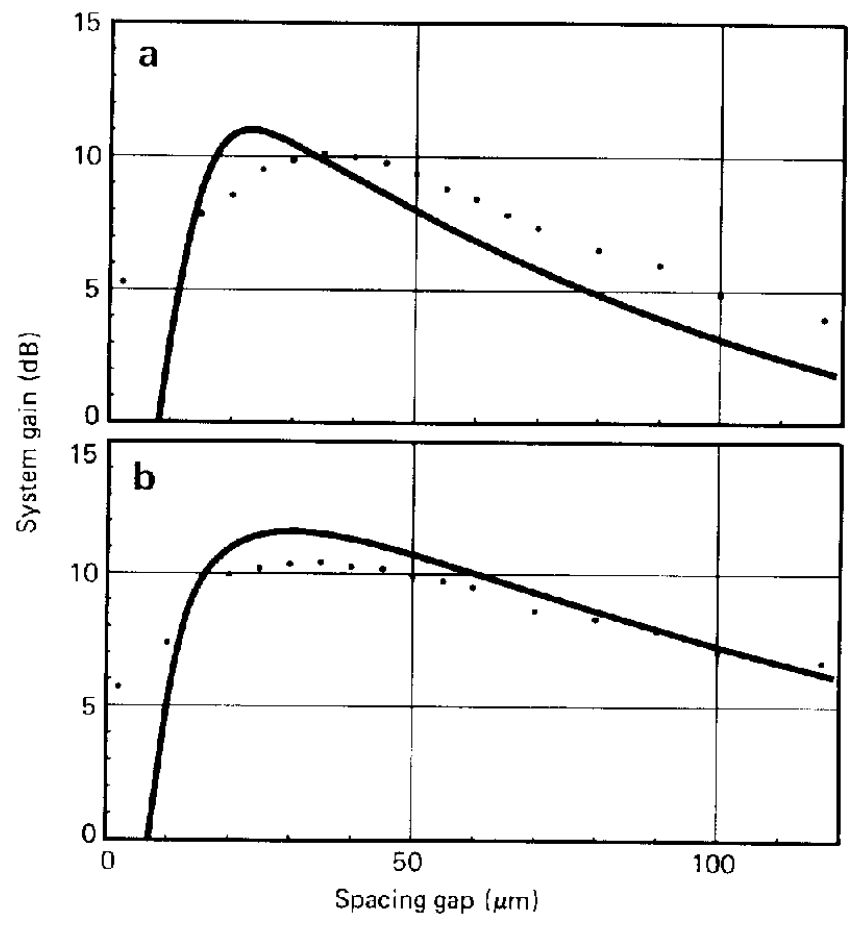

Figure 10 Measured sound pressure gain as a function of gap spacing for the design in Table 2 but with horn throat radii of (a) $0.18 \mathrm{~mm}$ and (b) $0.30 \mathrm{~mm}$. The curves show the behaviour calculated from the theory

direction to detect possible changes in the beam profile between the output with the mask and the output with the multi-horn plate altered some of the minor structure in the results but did not change the overall form. At low frequencies the response curve contained significant peak structure, which appeared to be extraneous to the actual quantity being measured. This is reflected as a wider scatter of data below $\approx 50 \mathrm{kHz}$.

Clearly the agreement between the measurements and the predictions of the theory is reasonably good, though not perlect. The most obvious possible source of error in the measurements lies in the imperfection of the matching surfaces on the plate and the transducer. both in relation to flatness and surface roughness. Repeated trials using different finishing techniques did not, however. result in improved agreement, and it is concluded that the fabrication techniques for the plates are adequately under control. The theory, however, does not claim to be completely accurate and a source of possible error lies in the treatment of wall effects in the spacing gap. In particular. the expansion used to include the imaginary part of the propagation vector $k$ in Equations (24) and (25) has only limited accuracy when the wall losses become high. and thus at low frequencies.

\section{Optimum spacing gap}

Measurements to determine the optimum spacing gap were all performed at $60 \mathrm{kHz}$ largely for reasons of convenience, since the transducer output at this frequency is high, the radial mode driving the transducer in the thickness direction by virtue of Poisson coupling. The apparatus has already been described and is shown in Figure 8. Measurements of the output were made at $5 \mathrm{~km}$ intervals, with a $2-3 \mu \mathrm{m}$ resolution, starting with the plate resting against the transducer face.

Figure 10 shows the measured results for the horn plate of Table 2, modified by surface grinding to give two different values of the horn throat diameter. Also shown superposed are curves giving the calculated behaviour. As predicted by the theory. the gain shows a broad maximum for a spacing of $\approx 30 \mu \mathrm{m}$, and the peak gain is a little more than $10 \mathrm{~dB}$. Most measured multi-horn plates performed in this way, but there were one or two cases of poor performance for very narrow throat diameters for which no explanation was found.

Several other features of the measurements warrant comment. In the first place, the agreement with experiment is again moderatcly satisfactory, although there is a discrepancy at low gap spacings. This can be explained in part by inaccuracy in the theory when wall effects in the gap are large, partly by the possibility of mechanical bridging between the plate and the transducer, and partly by pressure transmitted by bodily vibration of the matching plate as the axial impedance of the gap becomes higher. No allowance for this latter effect, which depends on the mechanical properties of the plate. is made in the theory. The sccond discrepancy is in the frequency and sharpness of the gain peak. Part of the lack of sharpness could be caused by defects in flatness of the matching plate, causing an effective averaging over neighbouring gap spacings. The fact that the disagreement is most severe for small throat diameters suggests, however, that there is a defect in either the theory of the throat geometry in these cases.

$\Lambda$ notable feature of both theory and measurement is the relative insensitivity of the gain to the radius of the horn throats, caused by approximate balance between the loss in horn gain and the improvement of coupling to the gap as the throat radius is increased. The same applies to the gap spacing, which could be set anywhere in the range $20-40 \mu \mathrm{m}$ with little effect on the gain. The lack of sensitivity in these two fine dimensions is clearly a great advantage when manufacture is considered.

\section{Conclusions}

The underlying concept and detailed design of the multi-horn plate described have been verified by the measurements. The measured performance is not in exact agreement with theoretical predictions, but is sufficiently close that no major uncertainties about the behaviour of the device remain. The theory is adequate to serve as a basis for design of matching plates for particular situations.

The matching plates developed here are able to provide gain of $\approx 10 \mathrm{~dB}$ over at frequency range from $\approx 30$ to $90 \mathrm{kHz}$. It is straightforward to scale the design to produce plates of higher or lower operating frequency, though theory indicates that the attainable performance may deteriorate somewhat if frequeneies much above $100 \mathrm{kH} z$ are involved.

The work described shows that it is possible to fabricate adequately precise multi-horn plates by hand in the laboratory and gives confidence that these devices could be produced simply, cheaply and to adequate tolerance in a manufacturing operation. The two fine dimensions. the separation gap between the multi-horn plate and the transducer surface, and the radius of the horn throat, are fortunately not critical in magnitude, so that simple assembly and testing techniques should be adequate.

\section{Acknowledgement}

The authors are most grateful to Dr Tony Collings for 
assistance with various aspects of horn-plate fabrication. The device is the subject of a patent application.

\section{References}

1 Morse, P.M. Vibration and Sound McGraw-Hill, New York (1948) reprinted American Institute of Physics, New York (1976) pp. 265-288

2 Olson, H.F. Acoustical Engineering Van Nostrand, Princeton (1975) pp. 100-123
3 Fletcher, N.H. and Thwaites, S. Obliquely truncated simple horns idealized models for vertebrate pinnae Acustica (1988) 65 194-204 4 Stevenson, A.F. Exact and approximate equations for wave propagation in acoustic horns $J$ Appl Phys (1951) $221461-1463$

5 Salmon, V. A new family of horns $J$ Acoust Soc Am (1946) 17 $212-218$

6 Benade, A.H. and Jansson, E.V. On plane and spherical waves in horns with nonuniform flare Acustica (1974) 31 79-98

7 Abramowitz, M. and Stegun, I.A. Handbook of Mathematical Functions National Bureau of Standards, Washington DC (1972) pp. $355-494$

8 Backus, J. Acoustic impedance of an annular capillary $J$ Acoust Soc Am (1975) 58 1078-1081 